\title{
Perfil de los adolescentes frente al consumo de tabaco de un colegio nacional del distrito de Puente Piedra 2012.
}

\author{
Durand-Aramburú Rocío ${ }^{1, a}$, Escate-Ruiz Lourdes ${ }^{2, a}$, Salvatierra-Goñe Edith ${ }^{3, a}$, \\ Cortez-Cuaresma Gloria ${ }^{2}$
}

\section{RESUMEN}

Objetivos: determinar el perfil de los adolescentes frente al consumo de tabaco del nivel secundaria del colegio nacional Fe y Alegría $\mathrm{N}^{\circ} 12$ del distrito de Puente Piedra. Material y métodos: estudio descriptivo de corte transversal, con una población de 424 alumnos de secundaria. Tras obtener el consentimiento firmado por los padres de familia aceptando la participación de sus hijos, se empleó como instrumento "Encuesta Global sobre el Uso del Tabaco entre la Juventud Peruana" elaborado y validado por la OMS, conformada por 14 preguntas, anónimo y auto aplicada. Resultados: se encontró que el 41,5\% de los adolescentes ha experimentado el consumo de tabaco aunque sea uno o dos piteadas. La edad de inicio del consumo con mayor prevalencia es de 12 - 13 años. El 10,14\% de los encuestados fumó 1 ó 2 días en los últimos 30 días y el 9,20\% refiere fumar 1 cigarrillo diario, teniendo como el lugar más frecuente de consumo a los eventos sociales (10,38\%). El 13,44\% de los encuestados consigue cigarrillos en una tienda, el $16,5 \%$ de los encuestados indica que su edad no fue impedimento para comprar cigarrillos, pero el 1,42\% refiere que una persona mayor se los facilita. De los adolescentes encuestados 1,40\% fuma más cuando bebe alcohol o usa otras drogas y un 4,01\% refiere sentir ganas de fumar apenas se despierta por la mañana. Conclusiones: existe un alto porcentaje de adolescentes que consumen tabaco, se debe mejorar la información sobre tabaco y salud en los colegios utilizando los medios de comunicación.

PALABRAS CLAVE: consumo, tabaco, perfil, adolescentes.

\section{Front profile of teenage snuff consumption of a national school in the District of Puente Piedra 2012.}

\section{SUMMARY}

Objectives: to determine the profile of adolescents to the consumption of snuff from the secondary school level National Fe y Alegria No. 12 district of Puente Piedra. Material and Methods: a descriptive cross-sectional study with a sample of 424 high school students from a national school district of Puente Piedra. After obtaining informed consent formed by parents to accept the participation of their children, the survey which was used as an instrument "Global Survey on the Use of Snuff between the Peruvian Youth" developed and validated by WHO, made was done by 14 questions, this is anonymous and self-applied. Results: we found that $41.5 \%$ of adolescents have experimented with snuff even one or two piteadas. The age of first use is most prevalent 12 to 13 years. 10.14\% of respondents smoked 1 or 2 days in the last 30 days and $9.20 \%$ reported daily cigarette smoking 1 , with the most common place where cigarettes are consumed social events $(10.38 \%)$ and one of the least frequent is school $(5.66 \%)$. The $13.44 \%$ of respondents get cigarettes in a store, $16.5 \%$ of respondents indicated that their age was not impediment to buy cigarettes, but the $1.42 \%$ reported that an elderly person is the easier. Of teens surveyed $1.40 \%$ smoke more when drinking alcohol or using other drugs and $4.01 \%$ refers feel like smoking just wake up in the morning. Conclusions: there is a high percentage of teens who use snuff, should improve information on snuff and health in schools using the media.

KEYWORDS: consumption, snuff, profile, adolescents.

Clínica Stella Maris. Lima, Perú.

Clínica Cayetano Heredia. Lima,Perú.

Clínica Ricardo Palma. Lima,Perú.

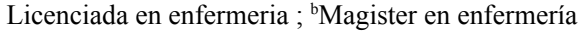




\section{INTRODUCCIÓN}

El tabaco contiene una sustancia llamada nicotina, esta es reconocida como adictiva por las diferentes organizaciones médicas internacionales. La nicotina cumple criterios fundamentales para poder decir que provocan adicción o dependencia, como por ejemplo los efectos psicoactivos originados a nivel del encéfalo que provocan un comportamiento denominado "efectos de refuerzo", este se manifiesta en las actitudes del consumidor, la adicción a la nicotina puede establecerse con gran rapidez en los adolescentes y jóvenes que empezaron a fumar recientemente (1).

El cigarrillo contiene más de 4700 sustancias químicas, varias de ellas, cancerígenas; una de las sustancias contenidas en el cigarrillo es la nicotina, la cual es de 6 a 8 veces más adictiva que la heroína (2).

La mayoría de los jóvenes que fuman de forma habitual, ya tienen una adicción a la nicotina. De hecho, ellos tienen la misma clase de adicción que tienen los fumadores adultos. De acuerdo con el Informe del Director General de Salud de 2012.

"De cada tres fumadores jóvenes, solo uno logrará dejar de fumar, y uno de los otros dos fumadores restantes morirá de causas relacionadas al tabaquismo. La mayoría de estos jóvenes nunca consideró las consecuencias a largo plazo sobre su salud asociadas con el consumo de tabaco cuando comenzaron a fumar; y la nicotina, una droga altamente adictiva, ocasiona que muchos continúen fumando en la edad adulta, a menudo con consecuencias mortales" (3). En el año 2003 se realizó el estudio de Tabaquismo en escolares de secundaria: Encuesta Mundial de Tabaquismo en jóvenes, cuyo objetivo principal fue documentar y monitorear la prevalencia del uso del tabaco, comprender y evaluar las actitudes, conocimientos y conductas de los estudiantes con relación al uso del tabaco y su impacto sobre la salud. Los datos recolectados hasta el año 2004 mostraron un panorama preocupante para las Américas (Global Tobacco Surveillance System Collaborating Group 2005). En nuestra región, la mitad de los adolescentes de 13 a 15 años de edad han fumado alguna vez en su vida. Para el mencionado estudio, se utilizó GYTZ una encuesta de corte transversal, la misma que fue utilizada en el presente trabajo (4).

Cada año mueren más de 40000 personas en el mundo a causa del tabaco, esta cifra oscila entre los cinco millones de muertes anuales y según el MINSA, en el Perú, mueren alrededor de 10 mil personas al año por enfermedades causadas por el humo de tabaco; por ello, actualmente el tabaquismo es considerado uno de los principales problemas de Salud Pública a nivel mundial(1).

Según proyecciones de la Organización Panamericana de la Salud, para el año 2030, el tabaco será la causa principal de muerte y discapacidad en todo el mundo, con más de 10 millones de víctimas mortales al año (2).

Adicionalmente a esto, en el Perú, el hábito de fumar comienza habitualmente en la adolescencia, debido a diversos factores de tipo sociocultural y personales que conducen a muchas personas a las pruebas iniciales del cigarrillo; que en muchos casos deviene en adicciones más o menos severas, con las consecuencias conocidas (5).

El tabaco está dejando de ser una droga social en muchos ambientes y su uso legal viene siendo restringido ya que está prohibido fumar en lugares públicos y hacer propaganda del tabaco durante el día, sin embargo, la propaganda abierta y encubierta está dirigida al consumo precoz de los jóvenes, quienes vienen fumando cada vez más, el hecho que el consumo no produzca cambios importantes en el comportamiento y que la toxicidad se manifieste al cabo de varios años de uso intensivo lleva a que no se aprecie con facilidad el peligro de su uso y que se perciba como una sustancia «fácil de dejar».

Las pocas investigaciones realizadas en el país respecto al uso de tabaco en adolescentes es el motivo principal para desarrollar el presente trabajo, ya que para muchos adolescentes experimentar a través del uso de drogas psicoactivas, representa un rito obligado al atravesar por dicha etapa, muchas veces influenciados por sus pares (6).

La adolescencia según la Organización Mundial de la Salud (OMS) es la etapa que transcurre entre los 10 y 19 años, considerándose dos fases, la adolescencia temprana 10 a 14 años y la adolescencia tardía 15 a 19 años (7). En cada una de las etapas se presentan cambios tanto en el aspecto fisiológico (estimulación y funcionamiento de los órganos por hormonas, femeninas y masculinas), cambios estructurales anatómicos y modificación en el perfil psicológico y de la personalidad; sin embargo, la condición de la adolescencia no es uniforme y varía de acuerdo a las características individuales y de grupo.

En el Perú una de las pocas investigaciones a nivel Nacional se realizó en el año 2005 donde se aplicó una encuesta global llamada GYTS adaptada a la juventud peruana.

Los resultados sorprendieron al encontrarse que la edad de inicio de uso de tabaco es los 13 años y que el consumo general en los escolares en estos últimos años ha aumentado (4). 
Además, según el Ministerio de Salud (Minsa), tres de cada diez escolares fuman cigarros, sin que sus padres conozcan de este hecho. Esta es una cifra que va en ascenso dentro de esta población pese a las medidas adoptadas, advierte el especialista de Cedro, Rojas $\mathrm{M}$, quién trabaja de cerca con adolescentes y jóvenes que han llegado al punto de sufrir adicciones (8).

La motivación para la presente investigación surge de los informes que viene siendo presentado por las diferentes instituciones de salud como la OMS, MINSA, etc. con respecto al elevado índice de adolescentes que inicia el consumo de tabaco. Esta situación nos lleva a reflexionar acerca de lo que ocurre en nuestro entorno social que induce al adolescente a que inicie el hábito de fumar, logrando muchas veces con esto a que se convierta en una adicción.

\section{MATERIAL Y MÉTODOS}

El presente estudio es de tipo descriptivo, porque estuvo dirigido a determinar el perfil de los adolescentes frente al consumo de tabaco y de corte transversal porque se recolectó la información en un solo momento, haciendo el corte en un tiempo. Se llevó a cabo en la institución Educativa Nacional Fe y Alegría N ${ }^{\circ} 12$ ubicado en el Distrito de Puente Piedra.

Se trabajó con la población total, que estuvo conformada por 424 alumnos (de 1ero a 5to año, siendo del 1er año 82 alumnos, 2do año 88 alumnos, 3ero año 86 alumnos, 4to año 90 alumnos y 5 to año 78 alumnos) del nivel secundaria del Colegio Fe y Alegría N ${ }^{\circ} 12$ del distrito de Puente Piedra, se tuvieron en cuenta los siguientes criterios de inclusión: estar matriculado en el año académico 2012, ser alumno(a) de 1 ero a 5to de secundaria, alumnos cuyos padres han firmado el consentimiento informado. No se incluyó a los alumnos que no querían participar.

Se tomó como referencia la "Encuesta Global sobre el Uso del Tabaco entre la Juventud Peruana" GYTS 2007 que fue elaborada por la OMS y modificada en Perú por Zavaleta A, Salas M y Peruga A, quienes emitieron su autorización para poder usarla, la misma que estaba subdividida en 7 partes, de las cuales solo se utilizó las primeras 14 preguntas que corresponden a la sección llamada: "el uso del tabaco en los adolescentes en los últimos 30 días". Todas las encuestas tuvieron una duración máxima de 20 minutos y se realizaron entre el Lunes 10 al Viernes 14 de Diciembre del 2012.

En la fase previa a la recolección de datos, se solicitó la autorización a la Directora del Colegio Fe y Alegría $\mathrm{N}^{\circ} 12$ del distrito de Puente Piedra y se informó a los padres de los estudiantes sobre el objetivo del estudio, con la finalidad de que sus hijos puedan participar en la encuesta y se procedió a la firma de los consentimientos informados por parte de los padres y de los asentimientos informados por parte de los adolescentes.

El procesamiento de los datos recolectados se llevó a cabo a través del programa Excel versión 2010, la información recolectada se presentó mediante tablas. Para el análisis de los datos se utilizó la estadística descriptiva como el porcentaje.

\section{RESULTADOS}

Tabla 1. Prevalencia de los adolescentes que consumieron tabaco del colegio nacional fe y alegría ${ }^{\circ} 12$. Distrito de Puente Piedra.

Alguna vez ha fumado aunque sea solo una vez

\begin{tabular}{ccc} 
& $\mathbf{N}$ & $\mathbf{\%}$ \\
\hline SÍ & 176 & 41,5 \\
NO & 248 & 58,5 \\
Total & 424 & 100.0 \\
\hline
\end{tabular}

La tabla $\mathrm{N}^{\circ} 1$, muestra que el $41.5 \%$ de los adolescentes han fumado alguna vez en su vida.

Tabla 2. Edad de inicio de consumo de tabaco de los adolescentes del colegio nacional Fe y Alegría n ${ }^{\circ} 2$. Distrito de Puente Piedra.

\begin{tabular}{lrr}
\hline Edad de inicio de consumo de tabaco & $\mathrm{N}^{\circ}$ & $\%$ \\
\hline Nunca he fumado cigarrillos & 248 & 58,5 \\
7 años & 12 & 2,83 \\
$8-9$ años & 4 & 0,94 \\
$10-11$ años & 31 & 7,31 \\
$12-13$ años & 56 & 13,21 \\
$14-15$ años & 52 & 12,26 \\
16 años o más & 21 & 4,95 \\
Total & 424 & 100,0
\end{tabular}

La tabla 2 muestra que $12(2,83 \%)$ de los adolescentes menciona haber consumido tabaco a los 7 años, pero la edad de inicio del consumo de tabaco con mayor prevalencia es de 12 - 13 años que representa un $13,31 \%$. 
Tabla 3. Cantidad, frecuencia y lugar más frecuente del consumo de tabaco de los adolescentes del colegio nacional Fe y Alegría n ${ }^{\circ} 12$. Distrito de Puente Piedra

\begin{tabular}{llrr}
\hline & & $\mathbf{N}^{\circ}$ & $\mathbf{\%}$ \\
\hline & Ningún día. & 343 & 80,90 \\
Número de días que & 1 ó 2 días. & 43 & 10,14 \\
fumó cigarrillos & De 3 a 5 días. & 5 & 1,18 \\
durante los últimos & De 6 a 9 días. & 19 & 4,48 \\
30 días. & De 10 a 19 días. & 2 & 0,47 \\
& De 20 a 29 días. & 8 & 1,89 \\
& Todos los días. & 4 & 0,94 \\
\hline & No he fumado cigarrillos durante los últimos 30 días. & 343 & 80,90 \\
Número de cigarri- & Menos de un cigarrillo diario. & 17 & 4,01 \\
llos fumados al día & 1 cigarrillo diario. & 39 & 9,20 \\
durante los últimos & De 2 a 5 cigarrillos diarios. & 15 & 3,54 \\
30 días. & De 6 a 10 cigarrillos diarios. & 7 & 1,65 \\
& De 11 a 20 cigarrillos diarios. & 1 & 0,23 \\
& Más de 20 cigarrillos diarios. & 2 & 0,47 \\
\hline & Nunca he fumado cigarrillos. & 248 & \\
& En mi casa. & 18 & 4,49 \\
& En la escuela. & 24 & 4,25 \\
& En el trabajo. & 0 & 5,66 \\
Lugar donde fuma & En casa de amigos. & 23 & 5,42 \\
con frecuencia. & En eventos sociales. & 44 & 10,38 \\
& En otros espacios públicos (por ejemplo, parques, centros comerciales, & 37 & 8,72 \\
& esquinas). & 30 & 7,08 \\
& En cualquier lugar. & &
\end{tabular}

La tabla 3 muestra que el 10,14\% de los adolescentes fumó 1 ó 2 días en los últimos 30 días y el 0,94\% todos los días en los últimos 30 días. El 9.20\% refiere fumar 1 cigarrillo diario, el lugar más frecuente donde se consume cigarrillo son los eventos sociales $(10,38 \%)$ y uno de los menos frecuentes es en la escuela $(5,66 \%)$.

Tabla 4. Acceso al tabaco de los adolescentes del colegio nacional Fe y Alegría n ${ }^{\circ} 12$. Distrito de Puente Piedra

\begin{tabular}{|c|c|c|c|}
\hline & & $\mathbf{N}^{\circ}$ & $\%$ \\
\hline \multirow{9}{*}{$\begin{array}{l}\text { Lugar donde consigue } \\
\text { cigarrillos en los últimos } \\
30 \text { días. }\end{array}$} & & 343 & 80,90 \\
\hline & No he fumado cigarrillos durante los últimos 30 días. & 57 & 13,44 \\
\hline & Los compraba en una tienda. & 2 & 0,47 \\
\hline & Los compraba en una máquina de cigarrillos. & 5 & 1,18 \\
\hline & Le daba dinero a otra persona para que me los comprara. & & \\
\hline & Se los pedía a alguien o alguien me invitaba. & 7 & 1,65 \\
\hline & Los robaba. & 1 & 0,24 \\
\hline & Una persona mayor me los daba. & 6 & 1,42 \\
\hline & Los obtenía de otra manera. & 3 & 0,70 \\
\hline \multirow{3}{*}{$\begin{array}{l}\text { En los últimos } 30 \text { días al- } \\
\text { guien se negó a venderte } \\
\text { cigarrillos. }\end{array}$} & No he tratado de comprar cigarrillos durante los últimos 30 días. & 329 & 77,6 \\
\hline & Sí, alguien se negó a venderme. & 25 & 5,9 \\
\hline & No, mi edad no me impidió comprar cigarrillos. & 70 & 16,5 \\
\hline
\end{tabular}

La tabla 4 muestra que el 13,44\% de los adolescentes menciona que consigue cigarrillos en una tienda y el 1,42\% refiere que una persona mayor se los daba. El 16,5\% de los adolescentes indica que su edad no fue impedimento para comprar cigarrillos. 
Tabla 5. Deseo de fumar post ingesta de otras drogas y al despertarse de los adolescentes del colegio nacional Fe y Alegría ${ }^{\circ} 12$. Distrito de Puente Piedra

\begin{tabular}{llrr}
\hline & & $\mathbf{N}^{\circ}$ & $\mathbf{\%}$ \\
\hline \hline $\begin{array}{l}\text { Deseos de fumar después de haber } \\
\text { bebido alcohol o haber usado otra }\end{array}$ & Nunca he fumado cigarrillos. & 248 & 58,50 \\
droga. & Ya no fumo cigarrillos. & 128 & 30,19 \\
& Fumo pero nunca bebo alcohol o uso drogas. & 23,42 \\
& Fumo menos cuando bebo alcohol o uso drogas. & 5 & 1,18 \\
& Fumo más cuando bebo alcohol o uso drogas. & 1,40 \\
& Fumo casi lo mismo cuando bebo alcohol o uso & 6 & 3,30 \\
& drogas. & 248 & 58,50 \\
Apenas te despiertas por la maña- & Nunca he fumado cigarrillos. & 87 & 20,51 \\
na has fumado o sientes ganas de & Ya no fumo cigarrillos. & 71 & 16,74 \\
hacerlo. & No, no he fumado ni siento ganas de fumar apenas & 17 & 4,01 \\
& me despierto por la mañana. & 1 & 0,24 \\
& Sí, algunas veces siento ganas de fumar apenas me & 14 \\
& despierto por las mañanas. & Sí, siempre tengo ganas de fumar apenas me des- & 1 \\
& pierto por las mañanas. & \\
\hline \hline
\end{tabular}

La tabla 5 muestra que de los adolescentes, el 1,40\% fuma más cuando bebe alcohol o usa otras drogas y el $4,01 \%$ refiere sentir ganas de fumar apenas se despierta por la mañana.

\section{DISCUSIÓN}

En el Perú, el hábito de fumar comienza habitualmente en la adolescencia, debido a diversos factores de tipo sociocultural y personales que conducen a muchas personas a las pruebas iniciales del cigarrillo (5). El estudio que se ha desarrollado ha permitido la identificación de las características del consumo de tabaco entre los escolares de educación secundaria.

En cuanto al consumo de tabaco, se encontró que el 41,5\% de todos los estudiantes de secundaria han fumado tabaco alguna vez en su vida (Tabla 1). Haciendo la comparación con otros estudios, esta proporción es similar a la hallada por Aburto en Barcelona, España que menciona que el $51,6 \%$ han probado tabaco alguna vez en su vida (7) o como el 18\% de los adolescentes encuestados en América ya fuman habitualmente. Por eso se puede decir tanto en el Perú como en otros países el consumo de tabaco en adolescentes se está incrementando. Por otro lado, Garcia en su investigación menciona que se destaca que $12,7 \%$ de los adolescentes ha fumado al menos una vez en la vida y $9,0 \%$ fuma actualmente de un total de 1915 adolescentes encuestados (9).

En la tabla 2, se halló que el 2,8\% menciona haber consumido tabaco a los 7 años, pero la edad de inicio del consumo de tabaco con mayor prevalencia es de $12-13$ años, en similitud al estudio de Alcalá (1) el 31,9\%, con una media de comienzo del hábito tabáquico de 13,25 años. Por otro lado, Repetto menciona en su investigación que el promedio de edad en el cual probaron cigarrillos fue de 12,19 años en Chile (10). Por lo cual, podemos decir que 13 años es la edad más frecuente del inicio de consumo de tabaco.

En cuanto a la frecuencia, cantidad y lugar de consumo de tabaco de los adolescentes dentro del último mes, se encontró que la mayoría de los que han fumado lo hicieron 1 ó 2 días y uno de los menores porcentajes de fumadores lo hizo todos los días, el consumo de tabaco está involucrando actualmente a más temprana edad, el mayor porcentaje de fumadores ha consumido 1 cigarrillo diario en el último mes; además, los eventos sociales son considerados para los adolescentes como el espacio donde se consume el tabaco en grandes cantidades, donde se dejan influenciar por amigos y sienten la libertad ya que no hay personas mayores que puedan evitarlo. Todo lo contrario a la escuela, que es uno de los menos frecuentes ya que esta cuenta con autoridades que prohíben el consumo de tabaco (Tabla 3), similares respuestas encontró Gonzalez en su estudio donde la mayoría refirió que fumaban a escondida de sus padres a pesar de que ellos tenían conocimiento del hábito (11).

En la tabla 4, se observa que el mayor porcentaje de fumadores consigue cigarrillos en una tienda $(13,44 \%)$, al igual que Pardo y Piñeros en su investigación en Colombia, encontraron que por lo menos, el $60 \%$ de los estudiantes de Bucaramanga, Cali y Manizales compraron cigarrillos en una tienda o supermercado (12). 
En la tabla 5 se observa que a pesar de que un mínimo porcentaje menciona fumar más cuando bebe alcohol o usa otras drogas, esto se considera alarmante ya que se puede entender que una droga induce a consumir otra llegando a ser más perjudicial para la salud; así mismo, el 4.01\% de los encuestados refiere sentir ganas de fumar apenas se despierte dando a entender que para estos alumnos este hábito ya está siendo un problema porque ya está generando dependencia; similares resultados encontró $\mathrm{Da}$ Silva en su estudio donde se asoció el consumo de tabaco y el de bebidas alcohólicas, siendo que, en el grupo de adolescentes que fuma es donde se encuentra el mayor porcentaje de encuestados que ingieren alcohol (13).

\section{REFERENCIAS BIBLIOGRÁFICAS}

1. Ministerio de Salud. Ambientes Libres de Humo de Tabaco. Lima: MINSA; 2011. (Citado el 20 de noviembre del 2015) Disponible en: http://www. minsa.gob.pe/portada/Especiales/2011/antitabaco/ index.htm.

2. Ministerio de salud. Ambientes libres de humo de tabaco; Perú: MINSA; 2015 (Citado el 20 de noviembre del 2015) Disponible en: http://www. minsa.gob.pe/portada/Especiales/2015/sintabaco/ index.html

3. American Cancer Society. Datos sobre el uso del tabaco en los niños y adolescentes. American Cancer Society; 2015 (Citado el 20 de noviembre del 2015) Disponible en: http://www.cancer.org/espanol/ cancer/queesloquecausaelcancer/tabacoycancer/ fragmentado/datos-sobre-el-uso-del-tabaco-en-losninos-y-adolescentes-child-and-teen-tobacco-use

4. Zavaleta A, Salas M, Peruga A. Tabaquismo en escolares de secundaria: Encuesta Mundial de tabaquismo en jóvenes. Lima, Perú: MINSA; 2005. (Citado el 20 de noviembre del 2015) Disponible en: http://www.minsa.gob.pe/portada/ Especiales/2011/antitabaco/docsTrab/cuaderno15 tabaquismoescolaressecundaria.pdf

5. CEDRO. El problema de las Drogas en el Perú. Lima, Perú:CEDRO; 2015.

6. Rojas M. Consumo de drogas psicoactivas en un colectivo de púberes y adolescentes, pautas y tendencias. Lima, Perú: CEDRO; 2005.

7. Pineda S, Aliño M. El Concepto de Adolescencia. En: Pineda S, Aliño M. Manual de Prácticas clínicas para la atención integral de la Salud en la adolescencia. La Habana: MINSAP; 1999. p.15-23.(Citado el 20 de noviembre del 2015) Disponible en: http://aps.sld.cu/ bvs/materiales/manual_practica/Capitulo \%201.pdf

8. Ministerio de Salud. El Minsa alerta: tres de cada diez escolares fuman tabaco en el país. Lima, Perú: MINSA; 2015.
(Citado el 20 de noviembre del 2015) Disponible en: http://larepublica.pe/impresa/sociedad/3466-el-minsaalerta-tres-de-cada-diez-escolares-fuman-tabaco-enel-pais

9. García R, Varona P, Hernández M, Chang M, Bonet $\mathrm{M}$, García R. Influencia familiar en el tabaquismo de los adolescentes. Rev Cubana Hig Epidemiol. 2008; 46(3): (Citado el 20 de noviembre del 2015) Disponible en: http://scielo.sld.cu/scielo.php? script $=$ sci arttext\&pid=S1561-30032008000300009\&lng=es.

10.Repetto $\mathrm{P}$, Bernales M. ¿Cuántas personas fuman?: Percepción del nivel de consumo y su relación con el consumo de cigarrillos en adolescentes chilenos. Rev méd Chile. 2012; 140 ( 6 ): 740-745. (Citado el 20 de noviembre del 2015) Disponible en: http:// www.scielo.cl/scielo.php? script $=$ sci arttext\&pid=S0034-98872012000600007\&lng=es. http://dx.doi.org/10.4067/S0034-98872012000600007.

11. Gonzalez L, Berger K. Consumo de tabaco en adolescentes: factores $0 \mathrm{de}$ riesgo y factores protectores. Cienc. enferm. 2002 ; 8(2): 27-35. (Citado el 20 de noviembre del 2015) Disponible en: http://www. scielo.cl/scielo.php?script=sci_arttext\&pid $=$ S071795532002000200004\&lng=es. http://dx.doi. org/10.4067/S0717-95532002000200004

12.Pardo C, Piñeros M. Consumo de tabaco en cinco ciudades de Colombia, Encuesta Mundial de Tabaquismo en Jóvenes, 2007. Biomédica. 2010; 30(4): 509-518. (Citado el 20 de noviembre del 2015)Disponible en: http://www.scielo.org.co/scielo.php?script=sci_ arttext\&pid=S0120-41572010000400008\&lng=en

13.Ferreira M, Torgal M. Consumo de tabaco y alcohol en la adolescencia. Rev. Latino-Am. Enfermagem. 2010; 18(2):[08 pantallas]. (Citado el 20 de noviembre del 2015) Disponible en: http://www.scielo.br/pdf/ rlae/v18n2/es_17.pdf

\section{Correspondencia:}

Escate Ruiz Lourdes

Correo electrónico: lourdes.escate.r@upch.pe

Fecha de recepción: 22 de Octubre 2015

Fecha de aceptación: 02 de Diciembre 2015 